\title{
IMPACTO DE LAS REDES SOCIALES EN LOS INGRESOS DE LOS HOTELES EN COLOMBIA, ECUADOR Y PERÚ
}

\author{
JOAQUIM MAJÓ*, DAISSY MOYA** \& LAURA VALL-LLOSERA**** \\ UNIVERSITAT DE GIRONA
}

Recibido/ Received/ Recebido: 03/11/2016 - Aceptado/ Accepted/Aprovado: 16/11/2016

\begin{abstract}
Resumen
El objetivo de la presente investigación es analizar la influencia de las valoraciones de los hoteles en las redes sociales y cómo estas afectan los ingresos de los hoteles. A partir de los datos mensuales (entre agosto de 2014 y julio de 2015) de 48 hoteles de Colombia, Ecuador y Perú se analizaron principalmente las variables: GRI (Global Review Index), Oferta, Demanda, Venta, RevPAR (Revenue Per Avalable Room) y Ubicación; mediante análisis de conglomerados y un modelo lineal. Se encontró que un mejor GRI favorece a una mejor ratio entre las ventas y la oferta. En concreto, observamos que cuando el GRI se incrementa en una unidad, el RevPAR crece en 0,49\%, manteniendo el resto de variables constantes. Es la primera investigación realizada en Sudamérica para analizar la incidencia de las redes sociales en los ingresos de los hoteles.

Palabras clave: Redes sociales; Reputación Online; Satisfacción del huésped; Turismo; Re$v P A R$.
\end{abstract}

\section{IMPACT OF SOCIAL NETWORKS ON HOTELS' INCOMES IN COLOMBIA, ECUADOR AND PERU}

\begin{abstract}
The objective of this research is to analyze the influence of hotel ratings on social networks and how they affect hotel revenues. From 48 hotels` monthly data (between August 2014 and July 2015) of Colombia, Ecuador and Peru, the following variables were analyzed: GRI (Global Review
\end{abstract}

Decano Facultad de Turismo de la Universitat de Girona. Ingeniero en Informática de la Universitat Politècnica de Catalunya, Doctorado en Administración y Gestión de empresas. Miembro del Grupo de investigación: Laboratori Multidisciplinar de Recerca en Turisme. Dirección postal: ST. DOMĖNEC Plaça Ferrater Mora 117004 - GIRONA Despatx: B. Teléfono: +34 972419717.

Correo electrónico: joaquim.majo@udg.edu

* Docente Facultad de Administración de Empresas Turísticas y Hoteleras de la Universidad Externado de Colombia. Administradora de empresas Turísticas y Hoteleras de la Universidad Externado de Colombia, Especialista en Computación para la docencia de la Universidad Antonio Nariño, Especialista en Edumática con énfasis en Ludomática de la Universidad Autónoma de Colombia, y Especialista en Gerencia y Proyección Social de la Educación y Magíster en Ciencias de la Educación de la Universidad Libre de Colombia. Doctoranda en Turismo de la Universitat de Girona. Dirección postal: Carrera 88 ${ }^{a}$ No 21-75 casa 10 Bogotá. Teléfono de contacto: +57 (1) 3419900 Ext. 1408. Correo electrónico: daissy.moya@uexternado.edu.co

*** Docente Facultad de Economía de la Universitat de Girona. Licenciada en Economía y Doctora en Economía de la Universitat de Girona. Miembro del Grupo de investigación: Grup de Recerca en Estadística, Econometria i Salut (GRECS-UdG), Centro de Investigación biomèdica en Red en Epidemiologia y Salud (CIBERESP) y Compositional and Spatial Data Analysis (COSDA). Dirección postal: ECONOM/EMPRES Campus Montilivi 17003 - GIRONA Despatx: 218. Teléfono: +34 972418938 . Correo electrónico: laura.vall_llosera@udg.edu 
Index), Offer, Demand, Sale, RevPAR (Revenue Per Available Room) and Location; by conglomerates analysis and a linear model. It was found that a better GRI favors a better ratio between sales and supply. Specifically, we observe that when the GRI increases by one unit, the RevPAR grows by $0.49 \%$, keeping the rest of the variables constant. It is the first research conducted in South America to analyze the incidence of social networks on hotel revenues.

Keywords: Social networks; Online Reputation; Guest satisfaction; Tourism; RevPAR.

\section{IMPACTO DAS REDES SOCIAIS NOS RENDIMENTOS DOS HOTÉIS NA COLÔMBIA, NO EQUADOR E NO PERU}

\section{Resumo}

O objetivo da presente pesquisa é analisar a influência das avaliações dos hotéis nas redes sociais e como estas afetam seus rendimentos. A partir de dados mensais (entre agosto de 2014 e julho de 2015) de 48 hotéis da Colômbia, do Equador e do Peru, analisaram-se principalmente as variáveis: GRI (Global Review Index), oferta, demanda, venda, RevPAR (Revenue Per Avalable Room) e localização, mediante análise de conglomerados e um modelo linear. Encontrou-se que um melhor GRI favorece a uma melhor ratio entre as vendas e a oferta. Mais especificamente, observamos que quando o GRI se incrementa em uma unidade, o RevPAR cresce em 0,49\%, mantendo o resto de variáveis constantes. É a primeira pesquisa realizada na América do Sul para analisar a incidência das redes sociais nos rendimentos dos hotéis.

Palavras chave: Reputação on-line; Satisfação do hóspede; Turismo; RevPAR.

Majó, J., Moya, D. \& Vall-llosera, L. (2018) Impacto de las redes sociales en los ingresos de los hoteles en Colombia, Ecuador y Perú. En: Revista de la Facultad de Ciencias Económica: Investigación y Reflexión. rev.fac.cienc.econ, XXVI (1), DOI: https://doi.org/10.18359/rfce.3143

JEL: L83, M31, Z33.

\section{Introducción}

En la actualidad el ciberespacio brinda a los hoteleros nuevas estrategias para adquirir y retener huéspedes (Litvin, Goldsmith, \& Pan, 2008) y a los huéspedes nuevas formas de obtener información (Senecal \& Nantel, 2004) que les permite seleccionar entre tarifas, servicios, ubicación y calidad (Murphy \& Chen, 2014), entendiendo la calidad como la valoración expresada por los huéspedes en las redes sociales, OTAs (Online Travel Agencies) y sitios de opinión (Sigala, 2009) y por la valoración expresada en estrellas que otorgan estos sitios a los hoteles (Denizci Guillet \& Law, 2010).

Las reservas en el sector hotelero se encuentran influenciadas por la marca y los comentarios realizados por otros usuarios (Jun, Vogt, \& MacKay, 2010).
El presente estudio muestra el análisis realizado a 8 establecimientos de la cadena GHL Hoteles y 40 hoteles de su set competitivo ubicados en Colombia, Ecuador y Perú desde agosto de 2014 hasta julio de 2015 en el que mediante el uso de los programas Revinate y GHLink, se observó la oferta, demanda, valor de las ventas, RevPAR (Revenue per Available Room) y GRI (Global Review Index) mensual. Se analizaron también las variables tipo de hotel, ubicación y tiempo de utilización de canales de venta online.

Los hoteleros en América del Sur se preguntan qué relación hay entre el RevPAR y la valoración de los hoteles en las diferentes redes sociales, OTAs (Online Travel Agencies) y sitios de opinión. Teniendo en cuenta que ya se ha realizado un estudio en la Universidad de Cornell con información de Norte- 
américa (Anderson, 2012) y que en Sudamérica el comportamiento de los huéspedes suele ser diferente, se hace necesario realizar una investigación para demostrar dicha relación.

\section{Revisión de la literatura}

La información que incide en la toma de decisiones tanto de los turistas como de los administradores hoteleros se encuentra sistematizada y estudios recientes demuestran que la mayoría de las personas accede a ella desde dispositivos móviles (Fisher, 2016b), por ello planteamos un primer subcapítulo llamado Marketing Móvil. El segundo subcapítulo analiza las redes sociales más utilizadas actualmente y que influencian el proceso de compra de los huéspedes potenciales. En un tercer subcapitulo explicamos qué es la reputación online. En el cuarto subcapítulo, se presentan algunas estrategias para mejorar la reputación online y en el último subcapítulo, presentamos el software utilizado en la actualidad para medir la reputación online en los establecimientos hoteleros.

\subsection{Marketing móvil}

Los dispositivos móviles hoy por hoy están más presentes en nuestras vidas, se parecen cada día más a los computadores de escritorio (Al-Debei \& Al-Lozi, 2014), los usuarios se han convertido en testigos de estos avances tecnológicos haciendo que haya una competencia más reñida por parte de los proveedores de servicios de telecomunicaciones móviles (Seo, Ranganathan, \& Babad, 2008). La utilización de estos dispositivos ha generado el Mobile Data Service (MDS) que consiste en el análisis del desarrollo, uso y adopción de la tecnología móvil.

El estudio realizado por la Universidad de Ottawa, Canadá, demuestra que el estilo de compra del consumidor, la confianza por la marca y el precio son factores motivantes para incentivar la adquisición de smartphones (Persaud \& Azhar, 2012). Del mismo modo, el estudio cualitativo realizado por la Universidad Boğaziçi, Turquía, expone un nuevo modelo que identifica los motivos del consumidor para compartir su ubicación en redes sociales (LBSNSs por sus siglas, Location-Based Social Network Si- tes) debido a que ha aumentado su participación en estas convirtiendo estos sitios en lugares para evaluar ubicaciones y servicios prestados, para estar más involucrados con los consumidores al momento de realizar opiniones, abriendo un nuevo campo para el Marketing Móvil (Ramendra Singh, Yavuz, \& Toker, 2014). Conocer la ubicación de los clientes permite identificar el segmento al que este pertenece, siendo este valor necesario cuando se analiza el comportamiento de los usuarios que utilizan las redes sociales (Madi, Brashear Alejandro, \& Brashear Alejandro, 2016).

Estos servicios se presentan por medio de la celular media, que se refiere a la convergencia de Tecnologías de Comunicación Móviles y Servicios de Comunicación de Datos (Hong, Thong, Moon, \& Tam, 2008). Esta tecnología se puede utilizar para el comercio móvil que consiste en proporcionar productos y servicios que utilizan tecnologías inalámbricas para facilitar las actividades de comercio electrónico sin limitaciones de tiempo y lugar (San Martín, López-Catalán, \& Ramon-Jeronimo, 2012). El Ecosistema del Marketing Móvil está determinado por un número de componentes de tipo tecnológico, regulatorio, comercial, social y legal (Martínez \& Arango, 2012).

Un segmento importante de consumidores en el sector turístico son los Millennials, personas entre los 18 a los 35 años, que se caracterizan por utilizar el comercio móvil al momento de buscar y hacer reservas. Un estudio realizado por Sabre Hospitality muestra que el 55\% de los Millennials encuestados ha entrado a comprar desde un celular y un $21 \%$ lo ha hecho desde una Tablet (Fisher, 2016a). Se demostró que los dispositivos móviles son usados más para comprar que para reservar con resultados de $40 \%$ desde teléfonos y $13 \%$ en tablets (Fisher, 2016b).

\subsection{Redes sociales}

Las redes sociales permiten a los internautas compartir y discutir datos, en ellas se publica información multimedial (texto, audio, video e imagen) (Cortizo, Carrero, \& Gómez, 2011). Las más utilizadas actualmente son Twitter que cuenta con 320 
millones de usuarios activos y se encuentra en más de 35 idiomas (Twitter, 2016), Facebook con 1650 millones de usuarios en 110 idiomas (Facebook, 2016), Instagram con 300 millones de usuarios que publican aproximadamente 60 millones de fotos cada día (Instagram, 2016) y YouTube con mil millones de usuarios que se encuentra disponible en 76 idiomas (YouTube, 2016).

El sitio de opinión más importante del sector turístico y hotelero es TripAdvisor que a julio de 2016 contaba con 390 millones de visitantes únicos mensuales, 435 millones de opiniones sobre $6.8 \mathrm{mi}-$ llones de alojamientos, restaurantes y atracciones (TripAdvisor, 2016), este sitio presta servicio a los huéspedes que cada día se documentan más sobre el destino que van a visitar, buscando información online y creando una imagen previa que luego les permitirá gestionar las reservas ( $\mathrm{Li}$, Pan, Zhang, \& Smith, 2009).

Las nuevas tecnologías, afectan el conocimiento, las actitudes y el comportamiento de los turistas, debido a la transparencia que hay tanto de precios como de productos, esto hace que aumente el poder de los viajeros, que cada día son más sensibles a los precios, menos leales a la marca y más sofisticados (Jauhari, Dabas, \& Manaktola, 2007), muchos clientes dependiendo de su cultura están empezando a reservar con anticipación y a aprovechar promociones online para conseguir mejores tarifas (Beldona \& Kwansa, 2008), están dejando atrás la intermediación de los llamados agentes de viaje face to face y están optando por las OTAs (Grønflaten, 2009).

Las redes sociales se han convertido en herramientas de marketing masivas (Hoffman \& Novak, 2012) donde los usuarios de internet pasan la mayor parte del tiempo (Lipsman, Mudd, Rich, \& Bruich, 2012). El uso de la tecnología ha cambiado la percepción de la calidad, la satisfacción y las intenciones de regresar al hotel (Berezina, Cobanoglu, Miller, \& Kwansa, 2012). Estos factores modifican la nueva forma de interactuar entre los compradores y vendedores (Liang \& Turban, 2011).

Estudios demuestran que las redes sociales inciden en los comportamientos de compra de esta década (Wi- lliams, Crittenden, Keo, \& McCarty, 2012; Xiang \& Gretzel, 2010) las empresas de hoy buscan aumentar sus clientes y mejorar su presencia en ellas (Ruzic, Andrilic, \& Ruzic, 2011). Las redes sociales y las tecnologías de la información y la comunicación (TICs) han revolucionado el sector hotelero modificando las actividades comerciales y la forma de promocionar sus servicios (Cohen \& Olsen, 2013).

\subsection{Reputación online}

Con la aparición de los sitios de opinión, toman importancia los comentarios basados en el conocimiento de otros usuarios de los servicios (Park \& Kim, 2009). Estudios demuestran que encontrar una calificación de una estrella es más impactante que encontrar una calificación de 5 estrellas (Chevalier \& Mayzlin, 2006; Willemsen, Neijens, Bronner, \& de Ridder, 2011), dichos comentarios aparecen en tres tipos de canales a saber: editoriales, de marketing o personales (Dickinger, 2010). Los comentarios editoriales que contienen logos, inspiran mayor credibilidad en los huéspedes potenciales (Sparks, Perkins, \& Buckley, 2013) también son considerados editoriales los blogs de viajes (Akehurst, 2009).

Los comentarios personales son analizados por los clientes que evalúan varios escritores y les permite tomar decisiones debido a la confianza que también generan las críticas de otros usuarios (Ayeh, Au, \& Law, 2013), especialmente las recibidas en las OTAs (Online Travel Agencies) debido a que para poder registrar las opiniones, debe haber utilizado los servicios y haber hecho la reserva por medio de ese canal de comercialización (Martínez, Bernal García, \& Mellinas, 2012).

Las opiniones recibidas, deben ser manejadas con todo el equipo de trabajo (Lee, Park, \& Han, 2008), deben ser tratadas de forma diferente en cada uno de los departamentos del hotel (Zhu \& Zhang, 2010) aprovechando los comentarios positivos (Torres \& Kline, 2013) que demuestran satisfacción por parte del huésped y teniendo especial cuidado con los negativos (Lee et al., 2008).

Los hoteleros están aprovechando las tecnologías de la información y la comunicación para diseñar 
sus estrategias de mercadeo porque Internet mejora la accesibilidad, comodidad, rapidez, novedad, $\mathrm{co}^{-}$ bertura global, riqueza de la información, flexibilidad, reducido coste y fácil acceso por parte de los huéspedes desde cualquier lugar del mundo y desde cualquier dispositivo (Morales, Agüera, \& Cuadra, 2015), la buena utilización de los comentarios, permite a los empresarios diseñar nuevas estrategias de marketing (Chen \& Xie, 2008) incluidas las nuevas estrategias ecológicas (SW Chan, 2013) que se basan en la óptima promoción de los servicios del hotel a través de su página web (Hsieh, 2012) observando siempre que los hoteles menos conocidos son los más analizados por los huéspedes en los sitios de opinión (Vermeulen \& Seegers, 2009), que existen escritores de opiniones que son más influenciadores que otros (Cheung, Lee, \& Rabjohn, 2008; Gretzel \& Yoo, 2008), que los comentarios encontrados inciden directamente en la compra online (Chatterjee, 2001; Davis \& Khazanchi, 2008), que la buena utilización de las redes sociales permite llegar a millones de usuarios en el mundo interesados en nuestros servicios (Kaplan \& Haenlein, 2010) y que la buena utilización de los comentarios registrados a nuestra empresa y a la competencia permiten diseñar productos y servicios diferenciadores de mejor calidad cada día (Clemons, Gao, \& Hitt, 2006).

Thakran \& Verma (2013) opinan que la presencia online no debe limitarse a los canales de comercialización, sino que además debe aparecer información en las redes sociales, en los sitios de opinión y tratar de tener el hotel posicionado en los principales buscadores. Entre tanto Baloglu et al. (2010) destacan la importancia de los sitios comparadores de precios que también son utilizados por los clientes antes de realizar una reserva online. Los hoteleros que administran eficientemente los canales de distribución y los precios, posicionan mejor su marca, fidelizan más a sus huéspedes y disminuyen el riesgo de perder clientes potenciales entre los intermediarios (O'Connor \& Murphy, 2008).

Existen factores que afectan el RevPAR (Revenue Per Available Room) (Sainaghi, 2011) tales como la ubicación, la calidad (Zhang, Ye, \& Law, 2011), la categoría, la tipología, la pertenencia a una cadena hotelera (Hernández, Fuentes, \& Morini, 2012) o los comentarios publicados en la web (Anderson, 2012; Ye, Law, Gu, \& Chen, 2011). La suma de información de un establecimiento publicada en la red se conoce como Reputación online, es decir, "la construcción social alrededor de la credibilidad, fiabilidad, moralidad y coherencia que se tiene de una persona, ente, organismo, institución o empresa, etc. Por ello solo es parcialmente controlable por el sujeto, ya que se crea y recrea a partir de las percepciones que conforman un estado de opinión, consideración y valoración de otros" (Del Fresno, 2012, pp. 13-14). Estudios confirman que los clientes están dispuestos a pagar mayor cantidad de dinero por un bien o servicio que tiene mejor reputación online (Resnick, Zeckhauser, Swanson, \& Lockwood, 2006)

La reputación online en el sector hotelero es muy importante debido a que los productos ofrecidos son intangibles y no pueden ser evaluados antes de su consumo, incrementando la importancia de los comentarios de otras personas. Muchos productos turísticos y hoteleros se perciben como compras de alto riesgo, por lo que las opiniones del grupo son un aspecto importante en el proceso de decisión de compra. (Diana-Jens \& Rodríguez, 2015).

\subsection{Estrategias para mejorar la reputación online}

En el sector de la hospitalidad cuando se habla de reputación online, no se debe pensar en reaccionar en el momento. Se trata de aprovechar la información para realizar cambios inteligentes y mejorar la experiencia de los huéspedes del hotel. Aprovechando que la información es pública, se debe utilizar para tener una referencia sobre la competencia. ¿Qué están haciendo mejor que yo? ¿Qué estoy haciendo mejor que ellos? ¿Cómo me puedo destacar entre la multitud?

Se deben utilizar las menciones positivas para aprender más acerca de las expectativas de los huéspedes, utilizar el software que existe en el mercado para encontrar las áreas en las que se tiene la oportunidad de mejorar y tomar las decisiones de presupuestos basados en los comentarios en los que se manifiestan las necesidades de los huéspedes (Guacaran, 2016). 
Generalmente no son los gestos lujosos los que mencionan los viajeros en sus reseñas, sino los pequeños detalles, las sorpresas inesperadas y las atenciones especiales a cargo del personal. (Craig, 2013).

Daniel Craig (2013) también recomienda contestar siempre los comentarios en las redes sociales y en las OTAs. Respecto a la información que aparece en la página web, sugiere que se debe ser transparentes en los precios, en la calidad, en los servicios complementarios, en las fotos, en los videos y en todas las estrategias de marketing del hotel.

Una estrategia de marketing importante es la publicidad mediante el uso de video. En un mundo con constantes distracciones, el video capta la atención y cuenta la historia, no necesita palabras ni traducciones. El video es efectivo porque es difícil de falsificar, a diferencia de las descripciones y de las fotos. Antes de la compra, los viajeros quieren asegurarse que están tomando la decisión correcta y el video les muestra lo que pueden esperar (Ruffell, 2013).

ReviewPro (2015) plantea 8 estrategias para mejorar la reputación online:

- Proporcionar un servicio destacable. Es importante el compromiso imparable de llevar el servicio a la excelencia, hacerlo tan destacable que obligue a los huéspedes a hablar de él en una opinión.

- Ser fiel a la marca. Imaginar las historias que se quiere que los huéspedes compartan al irse del hotel y movilizar al personal para que entienda cuál es su papel para hacer que suceda.

- Ser honesto. Exagerar los puntos fuertes y omitir las realidades decepcionará a los huéspedes durante su estancia y seguramente no publicarán las buenas reseñas esperadas.

- Movilizar a todo el equipo. Es importante involucrar a todo el personal, compartiendo con ellos las reseñas y especialmente mostrando los comentarios en los que se les menciona.
- Ofrecer un gran valor. Los hoteles que reciben los mejores comentarios son los generosos con los extras, como el acceso a wifi, desayuno, upgrades o bebidas de cortesía. Cuando toman estas decisiones los hoteles, las incluyen en el rubro de gastos que conllevan un beneficio en su reputación online.

- Ponerle pasión cada día. Contratar empleados con una actitud positiva y las habilidades necesarias para trabajar como propietario o administrador directamente con los huéspedes.

- Centrarse en la hospitalidad tradicional. Identificar la experiencia del huésped como principal prioridad e implicar a todos los departamentos en el trabajo de pedir opiniones para analizar el sentimiento de los clientes y mejorar la calidad diariamente.

- Evitar la escalada. Evitar las opiniones negativas mediante la formación y otorgando poderes al personal para que identifiquen y resuelvan los problemas en el establecimiento antes que escalen hasta quejas online.

En cuanto a las pautas para contestar las opiniones en las OTAs, ReviewPro (2016a) sugiere:

- Responder en lo posible en menos de 48 horas.

- Siempre agradecer al huésped por tomarse el tiempo de escribir para mejorar la calidad.

- Tratar de responder en el idioma que escribió el cliente.

- Aclarar los malos entendidos.

- Explicar la forma en que se realizó el seguimiento en caso de haber recibido una queja.

- Invitar al huésped a regresar. 


\subsection{Software utilizado para medir la reputación online en la industria hotelera}

El Global Review Index (GRI) es la medida de la Reputación online para los hoteles. Todos los comentarios recibidos en las redes sociales, sitios de opinión y OTAs son evaluados con un software que estandariza las calificaciones, convirtiendo estrellas, círculos, caras felices, calificaciones de 1 a 5 o de 1 a 10; en calificaciones de 1 a 5 tanto para evaluar los departamentos y servicios del hotel, como para evaluar la competencia. Es importante tener un registro del nivel de satisfacción de los clientes, en función de controlar la reputación online (Chun Rosa, 2005). Actualmente existen varias empresas que ofrecen software especializado en medir la reputación online para el sector hotelero, algunas de ellas son ReviewPro, captura los comentarios que los usuarios de las empresas turísticas y hoteleras registran en la web en más de 45 idiomas, en 161 webs de opinión y OTAs, analiza más de 18 millones de comentarios diariamente a más de 19 mil clientes en 110 países ( $R e$ -
viewPro, 2016b); Revinate se dedica a capturar, medir y optimizar la experiencia de los huéspedes combinando todas las reseñas online y menciones en redes sociales en una única visión integrada, tiene más de 28 mil clientes en 160 países (Revinate, 2016). Olery permite analizar los comentarios de hoteles, restaurantes y atractivos turísticos, su sede se encuentra en Amsterdam (Olery, 2016). ThrustYou fundada en 2008 por Benjamin Jost y Jakob Riegger, permite organizar los comentarios de los hoteles y restaurantes, en la actualidad tiene más de 100 empleados de 22 países (TrustYou, 2016). MyHotel, es una plataforma de inteligencia de negocios que mezcla información de diversas fuentes tales como encuestas de satisfacción, OTA's y Benchmark de mercado para ayudar a los hoteleros a entender su negocio y entorno, al tiempo que gestionan la satisfacción de sus huéspedes, es la única empresa creada en Latinoamérica (MyHotel, 2016). En la tabla 1, se presenta una tabla comparativa de cada una de las empresas que presta servicios de análisis de reputación online, sede y características.

Tabla 1. Empresas que comercializan software para medir la Reputación online de los hoteles

\begin{tabular}{|l|l|l|l|l|l|}
\hline \multicolumn{1}{|c|}{ EMPRESA/CARACTERÍSTICAS } & \multicolumn{1}{|c|}{ REVINATE } & REVIEWPRO & \multicolumn{1}{c|}{ MYHOTEL } & TRUSTYOU & OLERY \\
\hline \multicolumn{1}{|c|}{ Sede } & Estados Unidos & \multicolumn{1}{|c|}{ España } & \multicolumn{1}{c|}{ Chile } & Reino Unido & Holanda \\
\hline Calificaciones por OTA & $\mathrm{X}$ & $\mathrm{X}$ & $\mathrm{X}$ & $\mathrm{X}$ & $\mathrm{X}$ \\
\hline Número de opiniones por OTA & $\mathrm{X}$ & $\mathrm{X}$ & $\mathrm{X}$ & $\mathrm{X}$ & $\mathrm{X}$ \\
\hline Calificaciones por departamento & $\mathrm{X}$ & $\mathrm{X}$ & $\mathrm{X}$ & $\mathrm{X}$ & $\mathrm{X}$ \\
\hline Calificaciones de la competencia & $\mathrm{X}$ & $\mathrm{X}$ & $\mathrm{X}$ & $\mathrm{X}$ & $\mathrm{X}$ \\
\hline Análisis semántico & $\mathrm{X}$ & $\mathrm{X}$ & $\mathrm{X}$ & $\mathrm{X}$ & $\mathrm{X}$ \\
\hline Análisis de sentimientos & $\mathrm{X}$ & $\mathrm{X}$ & $\mathrm{X}$ & $\mathrm{X}$ & $\mathrm{X}$ \\
\hline Análisis de precios de la competencia & & & $\mathrm{X}$ & & \\
\hline
\end{tabular}

Fuente: Elaboración de los autores

Como se puede observar en la tabla 1 , todas las empresas prestan los servicios necesarios para analizar la reputación online de los hoteles, la diferencia radica en los precios y servicios adicionales. El valor del software está asociado al número de habitaciones y cantidad de servicios que desee adquirir, si aspira conocer la información de la competencia, deberá pagar un cargo adicional por cada hotel que quiera observar.

El software utilizado para analizar el Global Review Index, fue Revinate debido a que es el software utilizado por la cadena hotelera GHL, que nos brindó la información para realizar la investigación. 


\section{Diseño metodológico}

Para el desarrollo de la presente investigación se planteó un objetivo general, 5 hipótesis, y se llevó a cabo un proceso de investigación que combinó distintas metodologías estadísticas de análisis de datos. Esta combinación entre diferentes herramientas nos permitió observar de distintas maneras una misma realidad para obtener un mejor conocimiento de los datos y contrastar determinadas hipótesis relacionadas con la estacionalidad de la demanda aprovechando la estructura del diseño muestral.

Por ello, en primer lugar, se utilizó el análisis de conglomerados, como herramienta descriptiva multivariante, para poder examinar las relaciones interdependientes entre el conjunto completo de las distintas variables de estudio para comprender la relación entre varios grupos de variables. Posteriormente, puesto que la utilización del análisis de conglomerados implica un conocimiento incompleto de la clasificación de los datos, y con el fin de contrastar los resultados y ofrecer conclusiones más elaboradas, se optó por complementar el análisis con un Modelo Lineal de Efectos Mixtos (LMM) con la variables RevPAR como dependiente y el resto de variables como explicativas, trabajando con la información anual de cada hotel a partir de la media aritmética de las variables numéricas recogidas. Finalmente, aprovechando que las variables analizadas fueron recogidas mensualmente, se realizó un análisis con un Modelo Lineal Generalizado con medidas repetidas (MLG-MR) para determinar la existencia de diferencias provocadas por el efecto estacional de la demanda.

\subsection{Hipótesis}

El principal objetivo de este artículo es analizar la influencia de algunas variables en los ingresos de los hoteles, en concreto ¿cómo la valoración de los huéspedes en las redes sociales modifica el comportamiento de la oferta y la demanda?

Para ello planteamos la siguiente hipótesis principal

H1 A mejor valoración de los huéspedes en las redes sociales, mayor es el ingreso del hotel.
Si esta hipótesis se corrobora, se analizará si otras variables explicativas modifican esta relación.

H2 La localización de los hoteles por país modifica significativamente la relación entre la valoración de los huéspedes en las redes sociales y los ingresos de los hoteles.

H3 La localización de los hoteles por ciudad modifica significativamente la relación entre la valoración de los huéspedes en las redes sociales y los ingresos de los hoteles.

H4 La localización de los hoteles por zona modifica significativamente la relación entre la valoración de los huéspedes en las redes sociales y los ingresos de los hoteles.

H5 La estacionalidad de la demanda modifica significativamente la relación entre la valoración de los huéspedes en las redes sociales y los ingresos de los hoteles.

\subsection{Metodología}

Desde el primero de agosto de 2014 hasta el 31 de julio de 2015 se observaron 48 hoteles de Colombia, Ecuador y Perú.

\subsubsection{Muestra}

Se seleccionaron 8 hoteles de la Cadena GHL y 40 hoteles de su set competitivo. En Colombia se analizaron el Hotel Hamilton Court y 5 hoteles de su set competitivo, el Hotel Sonesta Bogotá y 5 hoteles de su set competitivo, el Hotel Four Points by Sheraton de Medellin y 5 hoteles de su set competitivo y el GHL Hotel Sunrise de San Andrés con 5 hoteles de su set competitivo.

En Perú se analizaron el Sonesta Hotel El Olivar y 5 hoteles de su set competitivo, y el Hotel Sonesta Cusco con 5 hoteles de su set competitivo.

En Ecuador se observaron el Hotel Sheraton Quito y 5 hoteles de su set competitivo y el Hotel Howard Johnson de Guayaquil con 5 hoteles de 
su set competitivo. Para un total de 48 hoteles ubicados en Colombia, Ecuador y Perú. Se utilizó el programa GHLink de la cadena GHL para medir la oferta, demanda, Venta y RevPAR, también se utilizó el programa Revinate para analizar el GRI mensual.

Tabla 2. Variables analizadas para cada hotel y mes.

\begin{tabular}{|c|c|c|}
\hline Variable & Descripción & Categorías \\
\hline Ciudad & Ciudad dónde está ubicado el hotel & $\begin{array}{l}\text { Bogotá } \\
\text { Medellín } \\
\text { San Andrés } \\
\text { Cusco } \\
\text { Lima } \\
\text { Guayaquil } \\
\text { Quito }\end{array}$ \\
\hline Cadena & Pertenencia o no del hotel a la cadena GHL & $\begin{array}{l}\text { Si } \\
\text { No }\end{array}$ \\
\hline País & País donde está ubicado el hotel & $\begin{array}{l}\text { Colombia } \\
\text { Ecuador } \\
\text { Perú }\end{array}$ \\
\hline Tipología & Tipología de hotel & $\begin{array}{l}\text { Centro } \\
\text { Negocios } \\
\text { Vacacional }\end{array}$ \\
\hline Ubicación & Localización del hotel & $\begin{array}{l}\text { Zona Centro Ciudad } \\
\text { Zona Comercial - Negocios } \\
\text { Zona Turística }\end{array}$ \\
\hline Demanda & \multicolumn{2}{|l|}{ Número de habitaciones ocupadas } \\
\hline GRI & \multicolumn{2}{|l|}{ Global Review Índex } \\
\hline Oferta & \multicolumn{2}{|l|}{ Número de habitaciones disponibles } \\
\hline Meses & \multicolumn{2}{|c|}{ Número de meses que el hotel lleva operando en canales online } \\
\hline Venta & \multicolumn{2}{|c|}{ Ingresos recibidos por el hotel (en pesos colombianos) } \\
\hline RevPAR & \multicolumn{2}{|c|}{ Ingresos por habitación disponible (en pesos colombianos) } \\
\hline
\end{tabular}

Fuente: Elaboración de los autores

La información obtenida se analizó utilizando dos herramientas distintas. 
Por un lado, y con carácter exploratorio, se realizó un análisis de conglomerados o clúster, con el objetivo de obtener clasificaciones (clusterings) del conjunto de individuos en grupos homogéneos internamente y diferentes entre sí. Las variables de agrupación utilizadas fueron las variables cuantitativas demanda, GRI y RevPAR, todas estandarizadas dadas sus diferencias en las unidades de medida. Se utilizaron, como variables ilustrativas en este análisis, las variables cadena, zona, tipología, ciudad y país.

Por otro lado, se realizó un modelo lineal de efectos mixtos (LMM) (Rodríguez, 2007; Sainz \& González, 2004) con la variable RevPAR como variable dependiente y el resto de variables mencionadas anteriormente, como variables explicativas introduciendo las variables zona, país y ciudad como distintos niveles de agrupación para comprobar la influencia de la ubicación (según país, zona y ciudad) en la relación entre el GRI y el RevPAR. Para este modelo se trabajó con información anual de cada uno de estos hoteles a partir del cálculo de la media aritmética de cada una de las variables numéricas, como se expresa en la ecuación (1).

$$
y_{i j}=\alpha_{j}+\beta_{j} x_{i j}+e_{i j}
$$

Donde es el valor de la variable respuesta (RevPAR) de cada hotel (i) y para cada nivel de agregación ( $j$ ) (País, Zona o Ciudad); es el valor de la intercepta en el origen de coordenadas para cada nivel de agregación; es el valor particular de la pendiente de la recta para cada uno de los niveles de agrupación; es el valor de las variables explicativas; y es el error para cada hotel en cada nivel de agregación. En conjunto, se asume que su distribución sigue una distribución común normal con media cero y desviación típica (Bates, Mächler, Bolker, \& Walker, 2014; Seoane, 2014).

Puesto que los datos se recogieron mensualmente desde agosto de 2014 a julio de 2015, se ajustó, también, un modelo Lineal Generalizado con Medidas Repetidas (MLG-MR) (Arnau \& Bono, 2008; Leyland \& McLeod, 2000). Estos modelos permiten conocer no sólo los cambios o perfiles individuales, sino determinar si el cambio es significativo y si se dan diferencias entre los distintos sujetos de la muestra
(Raudenbush, 2001), posibilitándonos captar las posibles diferencias provocadas por el efecto estacional de la demanda, como se expresa en la ecuación (2).

$$
y_{i j}=\mu+\eta_{i}+\alpha_{j}+\varepsilon_{i j}
$$

Dónde es la observación $j$ del sujeto $i$; es una componente para todo $i$ y $j$, es el efecto aleatorio de sujeto; es el efecto fijo de un determinado momento del tiempo; y es un efecto aleatorio de error (ArnauGras, 2007).

Si bien las dos técnicas utilizadas tienen por objetivo la clasificación de los individuos, la diferencia fundamental entre ambas reside en que en el análisis de clúster los grupos son desconocidos a priori y son, precisamente, lo que queremos determinar. En cambio, en el análisis de regresión, los grupos son conocidos y lo que pretendemos es saber en qué medida las variables disponibles nos discriminan esos grupos y nos pueden ayudar a clasificar o asignar los individuos en los grupos dados.

Todos estos análisis se realizaron con el software estadístico R (Pinheiro \& Bates, 2016)

\section{Resultados}

\subsection{Análisis de conglomerados}

De los 48 hoteles analizados, 8 pertenecen a la cadena GHL. Una cuarta parte del total están ubicados en Bogotá y los restantes se encuentran repartidos, por partes iguales, en las ciudades de Cusco, Lima, Medellín, San Andrés Guayaquil, y Quito. El 75\% de estos hoteles se caracterizan por estar ubicados en zonas comerciales y de negocios, un $12,5 \%$ en zonas turísticas y otro $12,5 \%$ en zonas de centro ciudad.

En lo que refiere a la demanda y a la oferta, estos 48 hoteles registran una media anual de 3.296,50 y 4.590,14 habitaciones, respectivamente; con una venta media anual de \$799990.300. La media anual de meses que estos hoteles llevan operando en canales online es de 60,29. En lo que se refiere a la valoración medida por el Global Review Index (GRI), la media anual toma un valor de 4,23 puntos y el RevPAR asciende a una media de $\$ 172.250,50$. (Ver Imagen 1 ) 
Imagen 1. Dispersión anual RevPAR Vs.GRI

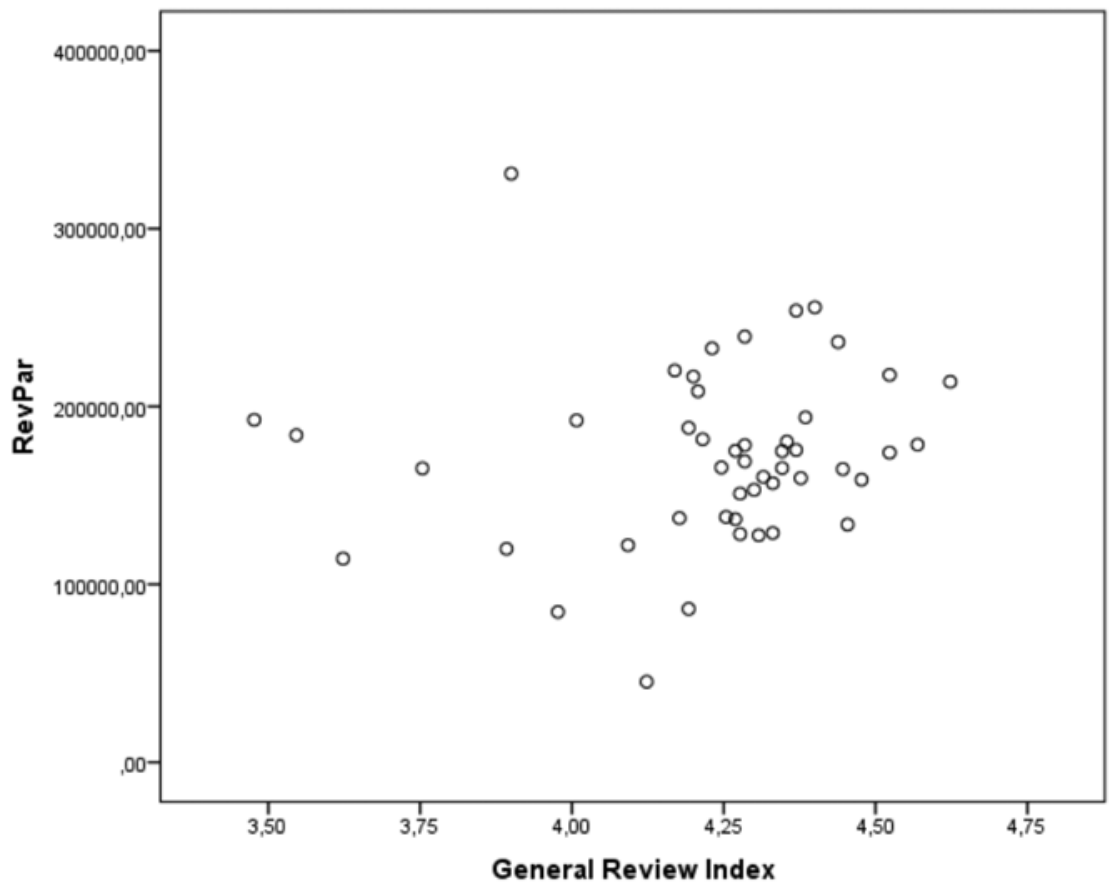

Fuente: Elaboración de los autores.

En relación al análisis de conglomerados, dadas las diferentes unidades de medida de las variables activas, se estandarizaron y se analizaron las correlaciones parciales sin que se observaran problemas de datos ausentes, de falta de linealidad o de valores atípicos. A partir del dendograma de "el vecino más próximo" se eliminan las observaciones atípicas del análisis.

La solución óptima obtenida con el método de agregación de Ward sugiere una agrupación de 4 clústers, con una $\mathrm{h}^{2}$ del $58,70 \%$ y con ningún grupo con una frecuencia inferior al $5 \%$ del total de la muestra.

Tabla 3. Medidas de asociación.

\begin{tabular}{|l|l|}
\hline & $\eta^{2}$ \\
\hline Zdemanda*Ward Method & 0,656 \\
\hline ZRevpar*Ward Method & 0,392 \\
\hline ZGRI*Ward Method & 0,713 \\
\hline
\end{tabular}

Fuente: Elaboración de los autores.
Tabla 4. Agrupación según el método de Ward.

\begin{tabular}{|l|l|l|}
\hline \multicolumn{1}{|c|}{ Clúster } & \multicolumn{1}{c|}{ Frecuencia } & \multicolumn{1}{c|}{ Porcentaje } \\
\hline 1 & 23 & 50,00 \\
\hline 2 & 11 & 23,90 \\
\hline 3 & 5 & 10,90 \\
\hline 4 & 7 & 15,20 \\
\hline Total & 46 & 100,00 \\
\hline
\end{tabular}

Fuente: Elaboración de los autores.

Los resultados del análisis de conglomerados con las variables activas (ZGRI, Zreupar y Zdemanda) y el análisis bivariante de los clústers con las variables ilustrativas (cadena, zona, tipología, ciudad y país) nos permiten definir el perfil de cada uno de los cuatro grupos. 
Tabla 5. Perfiles de los conglomerados según las variables activas e ilustrativas.

\begin{tabular}{ccccccc}
\hline Clúster & $\begin{array}{c}\text { Posición } \\
\text { demanda }\end{array}$ & $\begin{array}{c}\text { Posición } \\
\text { Revpar }\end{array}$ & $\begin{array}{c}\text { Posición } \\
\text { GRI }\end{array}$ & $\begin{array}{c}\text { Pertenece } \\
\text { cadena }\end{array}$ & Ubicación & Ciudad \\
\hline 1 & $4^{\mathrm{a}}$ & $2^{\mathrm{a}}$ & $1^{\mathrm{a}}$ & $\mathrm{Si}$ & Centro Ciudad & $\begin{array}{c}\text { Bogotá, Lima, } \\
\text { Cusco, Guayaquil }\end{array}$ \\
2 & $2^{\mathrm{a}}$ & $4^{\mathrm{a}}$ & $3^{\mathrm{a}}$ & $\mathrm{No}$ & Comercial y & Bogotá, Medellín \\
3 & $3^{\mathrm{a}}$ & $3^{\mathrm{a}}$ & $4^{\mathrm{a}}$ & $\mathrm{Si}$ & $\begin{array}{c}\text { Turística y Centro } \\
\text { Ciudad }\end{array}$ & $\begin{array}{c}\text { Medellín, San } \\
\text { Andrés, Cusco }\end{array}$ \\
4 & $1^{\mathrm{a}}$ & $1^{\mathrm{a}}$ & $2^{\mathrm{a}}$ & No & $\begin{array}{c}\text { Comercial y Negocios y } \\
\text { Centro Ciudad }\end{array}$ & $\begin{array}{c}\text { San Andrés, Lima, } \\
\text { Quito, Guayaquil }\end{array}$ \\
\hline
\end{tabular}

Fuente: Elaboración de los autores.

Esta clasificación nos permite ver que los grupos con un mejor GRI también tienen un mejor posicionamiento en el RevPAR. No se observa un patrón claro de relación entre el RevPAR y la pertenencia o no a la cadena, ni con la posición de la demanda, ni con la ubicación.

\subsection{Modelo Lineal de Efectos Mixtos (LMM)}

El modelo de regresión lineal con datos anuales es globalmente significativo al $1 \%$ y ofrece una bondad de ajuste ( $\mathrm{R}^{2}$ ajustado) del 48,34\%. A nivel individual son significativas algunas categorías de la variable ciudad (Lima, Quito y San Andrés) y el GRI. No es significativo el hecho de formar parte de la cadena, ni la demanda ni los meses que el establecimiento lleva presente en los canales online. Nótese que hemos obviado en este análisis de regresión las variables país, ubicación y tipología, por su correlación perfecta con la variable ciudad.

Los resultados de la tabla 6 muestran que cuando el GRI se incrementa en una unidad, el RevPAR crece en $0,49 \%$, manteniendo el resto de variables constantes. Del mismo modo, observamos que cuando un hotel está en Perú o en Ecuador su RevPAR es superior a un hotel ubicado en Colombia en un $0,57 \%$ y $0,24 \%$, respectivamente, independiente- mente de la valoración del GRI. Finalmente, cuando un hotel está ubicado en la zona turística presenta un RevPAR de 0,54\% superior a un hotel ubicado en la zona de negocios, independientemente de su GRI.

Tabla 6. Coeficientes modelo de regresión lineal múltiple con datos anuales.

\begin{tabular}{|l|l|l|l|}
\hline \multicolumn{1}{|c|}{ Variable } & & \multicolumn{1}{c|}{ ee } & \multicolumn{1}{c|}{ T } \\
\hline Incercepto & 9,548 & 0,7891 & $12,016^{\star \star *}$ \\
\hline Cadena.si & $9,698 \mathrm{e}^{-02}$ & $8,973 \mathrm{e}^{-02}$ & 1,081 \\
\hline Demanda & $1,584 \mathrm{e}^{-05}$ & $2,177 \mathrm{e}^{-05}$ & 0,728 \\
\hline GRI & $4,899 \mathrm{e}^{-01}$ & $1,737 \mathrm{e}^{-01}$ & $2,821^{\star}$ \\
\hline Meses & $2,887 \mathrm{e}^{-03}$ & $2,518 \mathrm{e}^{-03}$ & 1,146 \\
\hline Pais.Peru & $5.691 \mathrm{e}^{-01}$ & $1.130 \mathrm{e}^{-01}$ & $5.034^{\star \star *}$ \\
\hline Pais.Ecuador & $2.397 \mathrm{e}^{-01}$ & $9.080 \mathrm{e}^{-02}$ & $2.640^{\star}$ \\
\cline { 2 - 5 } Zona.Turística & $5.420 \mathrm{e}^{-01}$ & $1.344 \mathrm{e}^{-01}$ & $4.034^{\star \star *}$ \\
\hline Zona.Centro Ciudad & $-4.394 \mathrm{e}^{-01}$ & $1.352 \mathrm{e}^{-01}$ & $-3.249^{\star \star}$ \\
\hline
\end{tabular}

Signification codes: ${ }^{* * *} 0.001,{ }^{* *} 0.01,{ }^{*} 0.05$.

Fuente: Elaboración de los autores.

El análisis de regresión con efectos aleatorios para las variables país, ciudad y zona no presenta diferencias significativas en la relación entre el GRI y 
el RevPAR. Esto significa que la relación entre la valoración en los canales online por parte de los huéspedes y los ingresos de los hoteles no se ve modificada por la situación del hotel, ya sea en relación al país, a la ciudad o a la zona de ubicación.

\subsection{Modelo Lineal Generalizado con Medidas Repetidas (MLG-MR)}

Del mismo modo, el modelo Lineal Generalizado con Medidas repetidas (MLG-MR), es decir, considerando la fecha de recogida de los datos, no ofrece resultados estadísticamente significativos, constatando que no existen efectos estacionales en la demanda que afecten a la relación de las variables explicativas con el RevPAR.

\section{Conclusiones}

Tanto el análisis de conglomerados como la modelización a través de los LMM nos permiten concluir que un mejor GRI favorece a un mejor ratio entre las ventas y la oferta. En concreto, observamos que cuando el GRI se incrementa en una unidad, el RevPAR crece en $0,49 \%$, manteniendo el resto de variables constantes. Con ello podemos dar por contrastada nuestra hipótesis principal

H1 A mejor valoración de los huéspedes en las redes sociales, mayor es el ingreso del hotel.

En cuanto a las hipótesis secundarias observamos que para las variables país, ciudad y zona no presenta diferencias significativas en la relación entre el GRI y el RevPAR. Esto significa que la relación entre la valoración en los canales online por parte de los huéspedes y los ingresos de los hoteles no se ve modificada por la situación del hotel, ya sea en relación al país, a la ciudad o a la zona de ubicación. Por otra parte, la no significación del MLG-MR nos permite concluir que no existen efectos estacionales en la demanda que tengan efecto en la relación entre las variables explicativas y el RevPAR.

Con ello podemos refutar las hipótesis

H2 La localización de los hoteles por país modifica significativamente la relación entre la valora- ción de los huéspedes en las redes sociales y los ingresos de los hoteles.

H3 La localización de los hoteles por ciudad modifica significativamente la relación entre la valoración de los huéspedes en las redes sociales y los ingresos de los hoteles.

H4 La localización de los hoteles por zona modifica significativamente la relación entre la valoración de los huéspedes en las redes sociales y los ingresos de los hoteles.

H5 La estacionalidad de la demanda modifica significativamente la relación entre la valoración de los huéspedes en las redes sociales y los ingresos de los hoteles.

Por todo ello, como el RevPAR está afectado por la Reputación online, se deben tomar medidas para mejorar los comentarios que aparecen en las redes sociales, en las OTAs y en los sitios de opinión.

Se recomienda para futuras investigaciones aumentar el tamaño de muestra que permita ofrecer una información más completa de la situación real del país.

No queremos cerrar el documento sin indicar unas breves recomendaciones para mejorar y mantener una buena reputación online. En primer lugar, se recomienda implementar tácticas online y offline para incrementar las opiniones ya sea invitando a realizar comentarios en el momento del Check out o con acciones de estímulo con regalos promocionales. También es recomendable motivar a los huéspedes que han tenido experiencias agradables dentro del hotel a escribir su opinión en los sitios de opinión y las OTAs. En el caso de los huéspedes inconformes se deben identificar antes de su partida ofreciendo soluciones para evitar elevar el número de opiniones negativas.

En el momento de recibir una opinión positiva, es importante: felicitar a los empleados que brindaron su aporte para conseguirlo, contestar la opinión al huésped, destacar la opinión y socializarla con el equipo de trabajo e invertir en formación para conseguir un mayor número de opiniones positivas. 
Cuando se recibe una opinión negativa, lo primero que se debe hacer es agradecer al huésped por escribir, luego se debe disculpar con el huésped por la mala experiencia vivida, indicar cómo se realizará el seguimiento para evitar que se repita la situación, invitar al huésped a regresar al hotel para demostrarle que su experiencia fue un caso atípico.

En cualquier caso, todos los comentarios deben distribuirse a los departamentos afectados e incorporarse en la mejora permanente de la calidad. Se deben socializar entre todo el equipo buscando realizar cambios inteligentes para mejorar la experiencia de los huéspedes del hotel. Analizar también las opiniones de la competencia para orientar a los empleados a aprender más acerca de las expectativas de los huéspedes, utilizar el software que existe en el mercado para encontrar las áreas en las que se tiene la oportunidad de mejorar y tomar las decisiones de presupuestos basados en los comentarios en los que se manifiestan las necesidades de los huéspedes. Sin olvidar que los detalles mencionados por los viajeros en sus reseñas, son los más pequeños, las sorpresas inesperadas y las atenciones especiales a cargo del personal.

\section{Referencias}

Akehurst, G. (2009). User generated content: The use of blogs for tourism organisations and tourism consumers. Service Business, 3(1), 51-61.

Al-Debei, M. M., \& Al-Lozi, E. (2014). Explaining and predicting the adoption intention of mobile data services: A value-based approach. Computers in Human Behavior, 35: 326-338.

Anderson, C. (2012). The impact of social media on lodging performance. Cornell Hospitality Report, 12(15), 6-11.

Arnau, J., \& Bono, R. (2008). Estudios longitudinales de medidas repetidas: Modelos de diseño y análisis. Escritos De Psicología (Internet), 2(1), 32-41.

Arnau-Gras, J. (2007). Estudios longitudinales de medidas repetidas. modelos de diseño y de análisis. Avances En Medicin, 5, 9-26.

Ayeh, J. K., Au, N., \& Law, R. (2013). "Do we believe in TripAdvisor?" examining credibility perceptions and online travelers' attitude toward using user-generated content. Journal of Travel Research,, 0047287512475217.

Baloglu, S., Erdem, M., Brewer, P., Mayer, K., Christodoulidou, N., Connolly, D. J., \& Brewer, P. (2010). An examination of the transactional relationship between online travel agencies, travel meta sites, and suppliers. International Journal of Contemporary Hospitality Management, 22(7), 1048-1062.
Bates, D., Mächler, M., Bolker, B., \& Walker, S. (2014). Fitting linear mixed-effects models using lme4. arXiv Preprint arXiv: 1406.5823 ,

Beldona, S., \& Kwansa, F. (2008). The impact of cultural orientation on perceived fairness over demand-based pricing. International Journal of Hospitality Management, 27(4), 594-603.

Berezina, K., Cobanoglu, C., Miller, B. L., \& Kwansa, F. A. (2012). The impact of information security breach on hotel guest perception of service quality, satisfaction, revisit intentions and word-of-mouth. International Journal of Contemporary Hospitality Management, 24(7), 991-1010.

Chatterjee, P. (2001). Online reviews: Do consumers use them? Advances in Consumer Research, 28: 129-133

Chen, Y., \& Xie, J. (2008). Online consumer review: Word-ofmouth as a new element of marketing communication mix. Management Science, 54(3), 477-491.

Cheung, C. M., Lee, M. K., \& Rabjohn, N. (2008). The impact of electronic word-of-mouth: The adoption of online opinions in online customer communities. Internet Research, 18(3), 229-247.

Chevalier, J. A., \& Mayzlin, D. (2006). The effect of word of mouth on sales: Online book reviews. Journal of Marketing Research, 43(3): 345-354.

Chun, R. (2005). Corporate reputation: Meaning and measurement. Basingstoke: Palgrave Macmillan.

Clemons, E. K., Gao, G. G., \& Hitt, L. M. (2006). When online reviews meet hyperdifferentiation: A study of the craft beer industry. Journal of Management Information Systems, 23(2), 149-171.

Cohen, J. F., \& Olsen, K. (2013). The impacts of complementary information technology resources on the service-profit chain and competitive performance of south african hospitality firms. International Journal of Hospitality Management, 34: 245-254.

Cortizo, J. C., Carrero, F. M., \& Gómez, J. M. (2011). Introduction to the special issue: Mining social media. International Journal of Electronic Commerce, 15(3): 5-8.

Craig, D. (2013). 8 estrategias clave para mejorar tu puntuación en TripAdvisor | TecnoHotel. Recuperado de http://www. tecnohotelnews.com/2013/09/8-estrategias-clave-paramejorar-tu-puntuacion-en-tripadvisor/

Davis, A., \& Khazanchi, D. (2008). An empirical study of online word of mouth as a predictor for multi-product category ecommerce sales. Electronic Markets, 18(2), 130-141.

Del Fresno, M. (2012). El consumidor social. reputación online y social media Editorial UOC.

Denizci Guillet, B., \& Law, R. (2010). Analyzing hotel star ratings on third-party distribution websites. International Journal of Contemporary Hospitality Management, 22(6), 797-813.

Diana-Jens, P., \& Rodríguez, A. (2015). La reputación online y su impacto en la política de precios de los hoteles. Cuadernos De Turismo, (36), 129-155.

Dickinger, A. (2010). The trustworthiness of online channels for experience-and goal-directed search tasks. Journal of Travel Research, 
Facebook. (2016). Facebook. Recuperado de https://www.facebook.com/FacebookEspana/?brand_redir $=20531316728$

Fisher, H. (2016a). Is your hotel ready for millennials - and other device-savvy travelers? Sabre Hospitality Solutions Blog, Recuperado de http://sabrehospitality.com/resources/hotelmarketing-blogs/millennials-and-other-device-savvy-travelers

Fisher, H. (2016b). Millennials de-mystified: Who they are, how they shop, why they book. (). Retrieved from http://www2. sabrehospitality.com/millennials

Gretzel, U., \& Yoo, K. H. (2008). Use and impact of online travel reviews. Information and Communication Technologies in Tourism 2008: 35-46.

Grønflaten, Ø. (2009). Predicting travelers' choice of information sources and information channels. Journal of Travel Research, 48(2):230-244

Guacaran, A. (2016). 7 maneras de mejorar tu rendimiento en TripAdvisor. Recuperado de https://www.revinate.com/ blog/2016/03/7-maneras-de-mejorar-tu-rendimiento-entripadvisor/

Hernández, E., Fuentes, L., \& Morini, S. (2012). Una aproximación a la reputación en línea de los establecimientos hoteleros españoles. Papers De Turisme, (52), 63-88.

Hoffman, D. L., \& Novak, T. P. (2012). Toward a deeper understanding of social media. Journal of Interactive Marketing, 26(2), 69-70. Recuperado de http://www.econis.eu/ PPNSET?PPN=719146283

Hong, S., Thong, J. Y., Moon, J., \& Tam, K. (2008). Understanding the behavior of mobile data services consumers. Information Systems Frontiers, 10(4), 431-445.

Hsieh, Y. (2012). Hotel companies' environmental policies and practices: A content analysis of their web pages. International Journal of Contemporary Hospitality Management, 24(1), 97-121.

Instagram. (2016). Instagram. Recuperado de https://www. instagram.com/about/us/

Jauhari, V., Dabas, S., \& Manaktola, K. (2007). Managing reservations through online distribution channels: An insight into mid-segment hotels in india. International Journal of Contemporary Hospitality Management, 19(5), 388-396.

Jun, S. H., Vogt, C. A., \& MacKay, K. J. (2010). Online information search strategies: A focus on flights and accommodations. Journal of Travel \& Tourism Marketing, 27(6), 579-595.

Kaplan, A. M., \& Haenlein, M. (2010). Users of the world, unite! the challenges and opportunities of social media. Business Horizons, 53(1), 59-68.

Lee, J., Park, D., \& Han, I. (2008). The effect of negative online consumer reviews on product attitude: An information processing view. Electronic Commerce Research and Applications, 7(3), 341-352.

Leyland, A. H., \& McLeod, A. (2000). Mortality in england and wales, 1979-1992: An introduction to multilevel modelling using MLwiN. Glasgow: MRC Social and Public Health Sciences Unit,

Li, X. R., Pan, B., Zhang, L. G., \& Smith, W. W. (2009). The effect of online information search on image development:
Insights from a mixed-methods study. Journal of Travel Research, 48(1):45-57

Liang, T. \& Turban, E. (2011). Introduction to the special issue social commerce: A research framework for social commerce. International Journal of Electronic Commerce, 16(2): 5-14. doi:10.2753/JEC1086-4415160201

Lipsman, A., Mudd, G., Rich, M., \& Bruich, S. (2012). The power of "Like". Journal of Advertising Research, 52(1): 40-52.

Litvin, S. W., Goldsmith, R. E., \& Pan, B. (2008). Electronic wordof-mouth in hospitality and tourism management. Tourism Management, 29(3), 458-468.

Madi, A., Brashear Alejandro, T., \& Brashear Alejandro, T. (2016). Using values to segment virtual consumers on social networking sites. Marketing Intelligence \& Planning, 34(5)

Martínez, M., Bernal García, J. J., \& Mellinas, J. P. (2012). Los hoteles de la región de murcia ante las redes sociales y la reputación online. Revista De Análisis Turístico, (13), 1-10.

Martínez, O., \& Arango, P. (2012). El mobile marketing en colombia. su estado actual y proyección año 2012. Revista EAN, (73): 136-167.

Morales, P. M., Agüera, F. O., \& Cuadra, S. M. (2015). Análisis de las variables que influyen en la reputación online de las empresas turísticas. el caso de los hoteles de córdoba y granada. Gran Tour, Revista De Investigaciones Turísticas, (11): 103-120

Murphy, H. C., \& Chen, M. (2014). Online information sources used in hotel bookings examining relevance and recall. Journal of Travel Research,, 1-14. doi:0047287514559033

MyHotel. (2016). Conoce a myHotel - Nosotros. Recuperado de https://myhotel.com.es/nosotros/

O'Connor, P., \& Murphy, J. (2008). Hotel yield management practices across multiple electronic distribution channels. Information Technology \& Tourism, 10(2), 161-172.

Olery. (2016). Olery - Travel \& Hotel Data Specialist. Recuperado de http://www.olery.com/

Park, D., \& Kim, S. (2009). The effects of consumer knowledge on message processing of electronic word-of-mouth via online consumer reviews. Electronic Commerce Research and Applications, 7(4): 399-410.

Persaud, A., \& Azhar, I. (2012). Innovative mobile marketing via smartphones: Are consumers ready?. Marketing Intelligence \& Planning, 30(4): 418-443.

Pinheiro, P. \& Bates, D. (2016). R: Linear Mixed-Effects Models. Recuperado de https://stat.ethz.ch/R-manual/R-devel/library/ nlme/html/lme.html

Ramendra Singh, D., Yavuz, R., \& Toker, A. (2014). Location sharing on social networks: Implications for marketing. Marketing Intelligence \& Planning, 32(5): 567-585.

Raudenbush, S. W. (2001). Comparing personal trajectories and drawing causal inferences from longitudinal data. Annual Review of Psychology, 52(1), 501-525.

Resnick, P., Zeckhauser, R., Swanson, J., \& Lockwood, K. (2006). The value of reputation on eBay: A controlled experiment. Experimental Economics, 9(2), 79-101. 
ReviewPro. (2015). Guía | Las 8 Claves para Mejorar tu Ranking en TripAdvisor. Recuperado de https://www.reviewpro.com/ es/guia-estrategias-para-mejorar-ranking-tripadvisor-thanks/

ReviewPro. (2016a). Building a Winning OTA Strategy for Your Hotel. Recuperado de https://www.reviewpro.com/optimizehotel-performance-on-otas-thanks/

ReviewPro. (2016b). ReviewPro. Gestión de Reputación para Hoteles. Recuperado de https://www.reviewpro.com/es/ productos/gestion-de-reputacion-para-hoteles/

Revinate. (2016). Revinate Hospitality Management Software. Recuperado de https://www.revinate.com/company/about/

Rodríguez, A. A. (2007). Perspectiva de la construcción hotelera en el mediterrneo europeo. Anuario Jurídico Y Económico Escurialense, (40), 351-376.

Ruffell, C. (2013). Crucial Ideas for Effective Hotel Marketing, ReKnown. Recuperado de http://www.reelmarketer.com/ crucial-ideas-effective-hotel-marketing-reknown/

Ruzic, D., Andrilic, B., \& Ruzic, I. (2011). Web 2.0 promotion techniques in hospitality industry. International Journal of Management Cases, 13(4): 310-319.

Sainaghi, R. (2011). RevPAR determinants of individual hotels: Evidences from milan. International Journal of Contemporary Hospitality Management, 23(3), 297-311.

Sainz, R. C., \& González, G. M. (2004). Aplicación de los modelos mixtos a un caso práctico de modelización del crecimiento y producción de las masas forestales. Cuadernos De La Sociedad Española De Ciencias Forestales, (18), 317-321.

San Martín, S., López-Catalán, B., \& Ramon-Jeronimo, M. A. (2012). Factors determining firms' perceived performance of mobile commerce. Industrial Management \& Data Systems, 112(6), 946-963.

Senecal, S., \& Nantel, J. (2004). The influence of online product recommendations on consumers' online choices. Journal of Retailing, 80(2), 159-169.

Seo, D., Ranganathan, C., \& Babad, Y. (2008). Two-level model of customer retention in the US mobile telecommunications service market. Telecommunications Policy, 32(3): 182-196.

Seoane, J. (2014). ¿Modelos mixtos (lineales)? una introducción para el usuario temeroso. ETOLOGUÍA, 24: 15-37

Sigala, M. (2009). E-service quality and web 2.0: Expanding quality models to include customer participation and inter-customer support. The Service Industries Journal, 29(10), 1341-1358.

Sparks, B. A., Perkins, H. E., \& Buckley, R. (2013). Online travel reviews as persuasive communication: The effects of content type, source, and certification logos on consumer behavior Tourism Management, 39, 1-9.

SW Chan, E. (2013). Gap analysis of green hotel marketing. International Journal of Contemporary Hospitality Management, 25(7), 1017-1048.

Thakran, K., \& Verma, R. (2013). The emergence of hybrid online distribution channels in travel, tourism and hospitality. Cornell Hospitality Quarterly, 54(3), 240-247.

Torres, E., \& Kline, S. (2013). From customer satisfaction to customer delight: Creating a new standard of service for the hotel industry. International Journal of Contemporary Hospitality Management, 25(5), 642-659.

TripAdvisor. (2016). Acerca de TripAdvisor. Recuperado de https:// www.tripadvisor.es/PressCenter-c6-About_Us.html

TrustYou. (2016). TrustYou. Información general ·. Recuperado de http://www.trustyou.com/informacin-general?lang=es

Twitter. (2016). Red Social Twitter. Recuperado de https://about. twitter.com/es/company

Vermeulen, I. E., \& Seegers, D. (2009). Tried and tested: The impact of online hotel reviews on consumer consideration. Tourism Management, 30(1), 123-127.

Willemsen, L. M., Neijens, P. C., Bronner, F., \& de Ridder, J. A. (2011). "Highly recommended!" the content characteristics and perceived usefulness of online consumer reviews. Journal of Computer Mediated Communication, 17(1): 19-38.

Williams, D. L., Crittenden, V. L., Keo, T., \& McCarty, P. (2012). The use of social media: An exploratory study of usage among digital natives. Journal of Public Affairs, 12(2): 127-136. doi:10.1002/pa.1414

Xiang, Z., \& Gretzel, U. (2010). Role of social media in online travel information search. Tourism Management, 31(2): 179-188.

Ye, Q., Law, R., Gu, B., \& Chen, W. (2011). The influence of user-generated content on traveler behavior: An empirical investigation on the effects of $e$-word-of-mouth to hotel online bookings. Computers in Human Behavior, 27(2), 634-639.

YouTube. (2016). YouTube. Recuperado de https://www.youtube. $\mathrm{com} / \mathrm{yt} / \mathrm{press} / \mathrm{es} / \mathrm{statistics.html}$

Zhang, Z., Ye, Q., \& Law, R. (2011). Determinants of hotel room price: An exploration of travelers' hierarchy of accommodation needs. International Journal of Contemporary Hospitality Management, 23(7), 972-981.

Zhu, F., \& Zhang, X. (2010). Impact of online consumer reviews on sales: The moderating role of product and consumer characteristics. Journal of Marketing, 74(2), 133-148. 